\title{
Internal Rotation Analysis of the Ground State Microwave Spectrum of 2-Bromopropene
}

\author{
E. Fliege and H. Dreizler \\ Abteilung Chemische Physik im Institut für Physikalische Chemie der \\ Christian-Albrechts-Universität
}

Z. Naturforsch. 39a,637-645 (1984); received May 21, 1984

The microwave spectrum of 2-bromopropene was reinvestigated with the use of microwave Fourier transform spectroscopy. For the two isotopic species $\mathrm{CH}_{3} \mathrm{C}^{79} \mathrm{Br}=\mathrm{CH}_{2}$ and $\mathrm{CH}_{3} \mathrm{C}^{81} \mathrm{Br}=\mathrm{CH}_{2}$ the bromine quadrupole coupling was determined with higher accuracy. The barrier hindering internal methyl rotation was obtained from the ground state.

The microwave spectrum of 2-bromopropene, $\mathrm{CH}_{3} \mathrm{CBr}=\mathrm{CH}_{2}$, was initially investigated by Benz, Bauder and Günthard [1]. They assigned the spectrum in the ground and in the first excited state of the methyl torsion, and they determined the rotational and the bromine quadrupole coupling constants, and the barrier to internal rotation from the first excited torsional state.

By use of microwave Fourier transform (MWFT) spectroscopy $[2,3]$ it was possible to resolve the internal rotation doublets of high $J$ lines in the ground state and to determine the hindering potential $V_{3}$.

The substance was purchased from Ega Chemie, Steinheim, with $99 \%$ purity and used after vacuum distillation.

The spectra were taken at $-60{ }^{\circ} \mathrm{C}$ and pressures below $1 \mathrm{~m}$ Torr. One of the recordings is shown in Figure 1. The lines of the ${ }^{79} \mathrm{Br}$ - and ${ }^{81} \mathrm{Br}$-species measured in the frequency range from 8 to $18 \mathrm{GHz}$ are given in Tables 1 and 2.

In order to be able to extend the measurements to higher $J$-values, we performed an approximate centrifugal distortion analysis with the transitions of Table I and II of [1] and our own experimental frequencies just taking the centre of the quadruplet hfs pattern of each transition. The assignment was justified by the consistency of the experimental hfs patterns with a rough first order calculation using the quadrupole coupling constants given in [1].

For our final centrifugal distortion analysis with the Hamiltonian given by Van Eijck [4] and Typke

Reprint requests to Prof. Dr. H. Dreizler, Institut für Physikalische Chemie der Universität Kiel, Olshausenstr. 40, D-2300 Kiel.
[5] we had to constrict four of the quartic constants to zero. Otherwise a singular normal equation resulted in the fitting calculations. The best fit with a standard deviation of $175 \mathrm{kHz}$ for $\mathrm{CH}_{3} \mathrm{C}^{79} \mathrm{BR}=\mathrm{CH}_{2}$ and $124 \mathrm{kHz}$ for $\mathrm{CH}_{3} \mathrm{C}^{81} \mathrm{Br}=\mathrm{CH}_{2}$ was obtained with the choice of $\delta_{J}^{\prime}$ as fitting parameter besides the rotational constants. A similar observation is reported in [1]. As the centrifugal distortion analysis was used only for assignment, we did not try to improve the situation by more measurements.

The quadrupole hfs analysis was made by fitting $\chi_{+}=\chi_{b b}+\chi_{c c}$ and $\chi_{-}=\chi_{b b}-\chi_{c c}$ to the splittings of

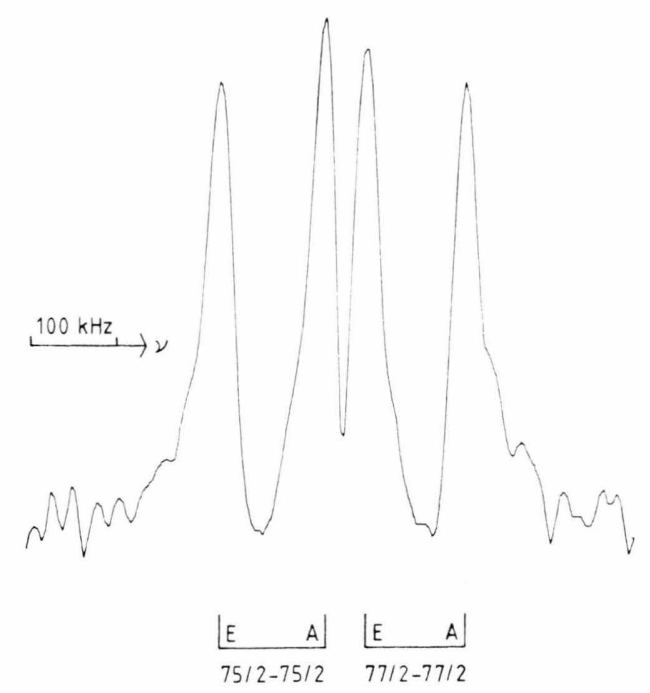

Fig. 1. A range of $700 \mathrm{kHz}$ out of a $10 \mathrm{MHz}$-scan of the rotational spectrum of $\mathrm{CH}_{3} \mathrm{C}^{81} \mathrm{Br}=\mathrm{CH}_{2}$ in natural abundance. $J_{K_{-} K_{+}}-J_{K^{\prime} K_{+}^{\prime}}^{\prime}=38_{9,29}-38_{9,30}$; amplitude spectrum, sample interval: $50 \mathrm{~ns}, 2048 \mathrm{~K}$ cycles, 1024 data points supplemented by 3072 zeros, microwave polarising frequency: $v_{\mathrm{MW}}=17449 \mathrm{MHz}$; pressure: $p=0.3 \mathrm{mTorr}$, temperature: $T=-59^{\circ} \mathrm{C}$. 
Table 1. Measured transitions of $\mathrm{CH}_{3} \mathrm{C}^{79} \mathrm{Br}=\mathrm{CH}_{2} . \Gamma$ : symmetry species, $v_{\text {exp }}$ : experimental frequency, $\bar{v}$ : arithmetic mean of the frequencies belonging to the same hfs transition and split by internal rotation, $\bar{v}_{0}$ : see text, $\bar{v}-\bar{v}_{0}$ : measured hfs shift, $\Delta v_{\mathrm{AE}}$ : experimental torsional splitting, $\Delta v_{\mathrm{sim}}$ : corrected torsional splitting, B: measured by Benz et al. [1], * : not used for quadrupole hfs analysis, nr: not resolved; frequencies, splittings and deviations in $\mathrm{MHz}$.

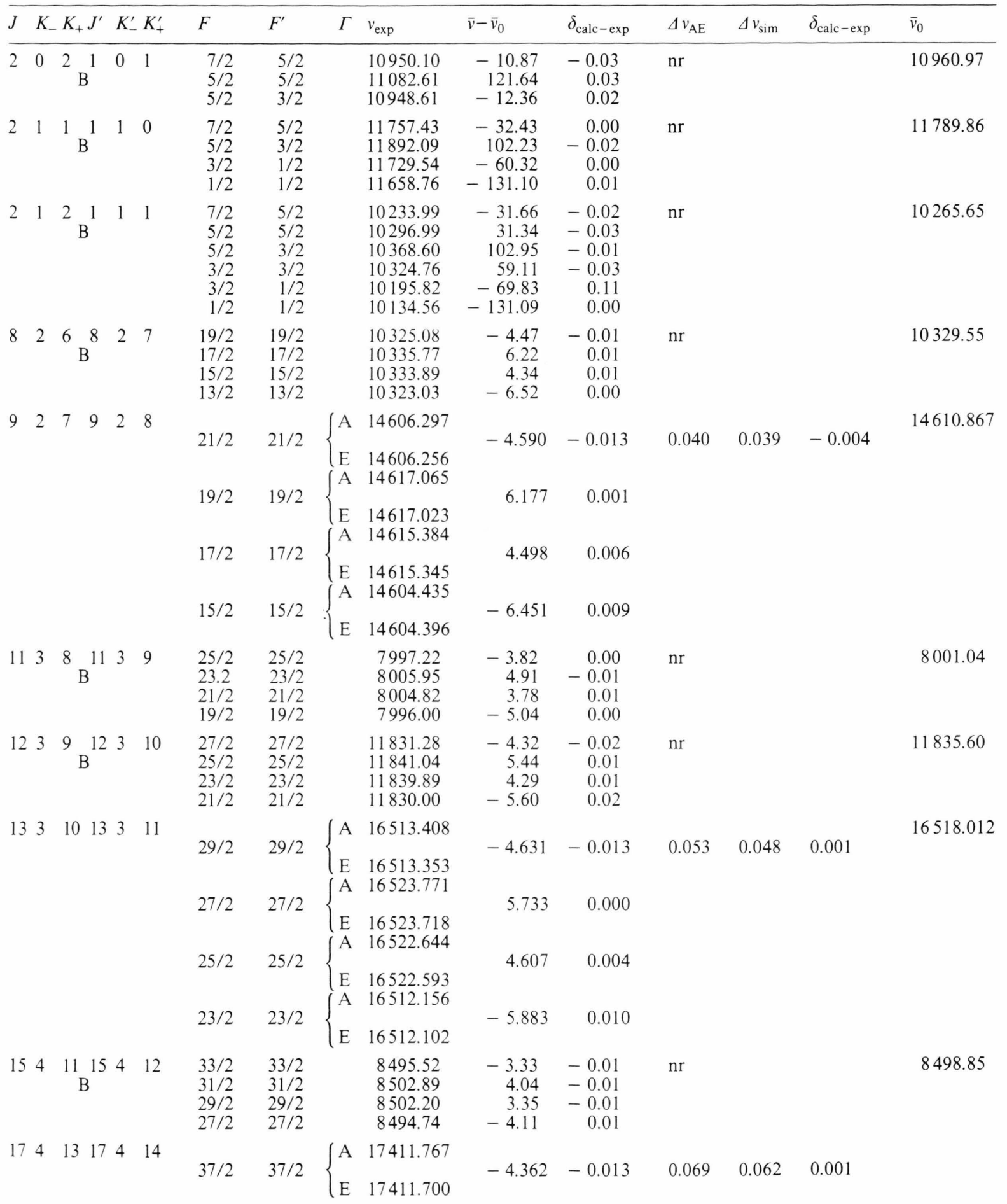


Table 1 (continued).

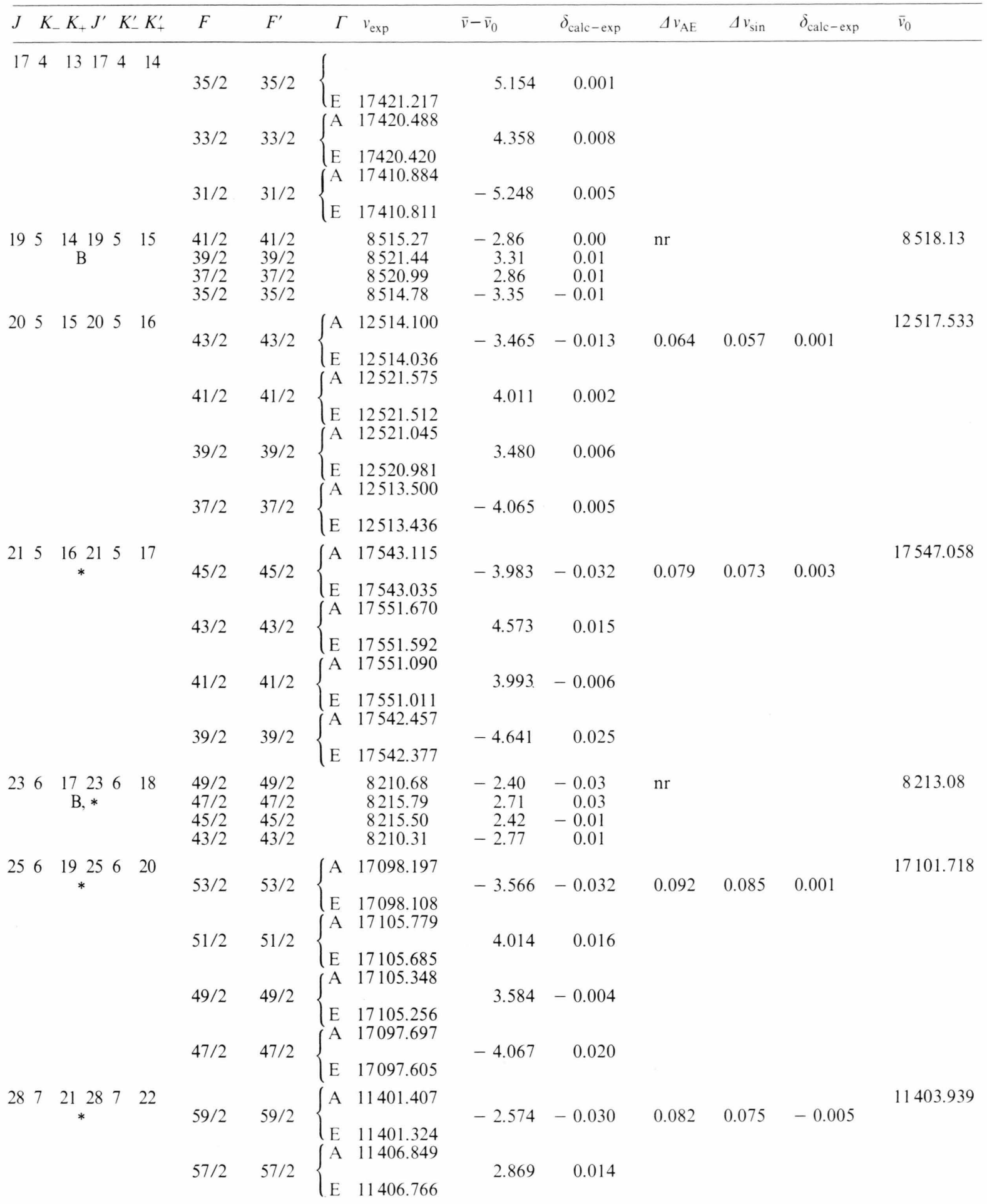


Table 1 (continued).

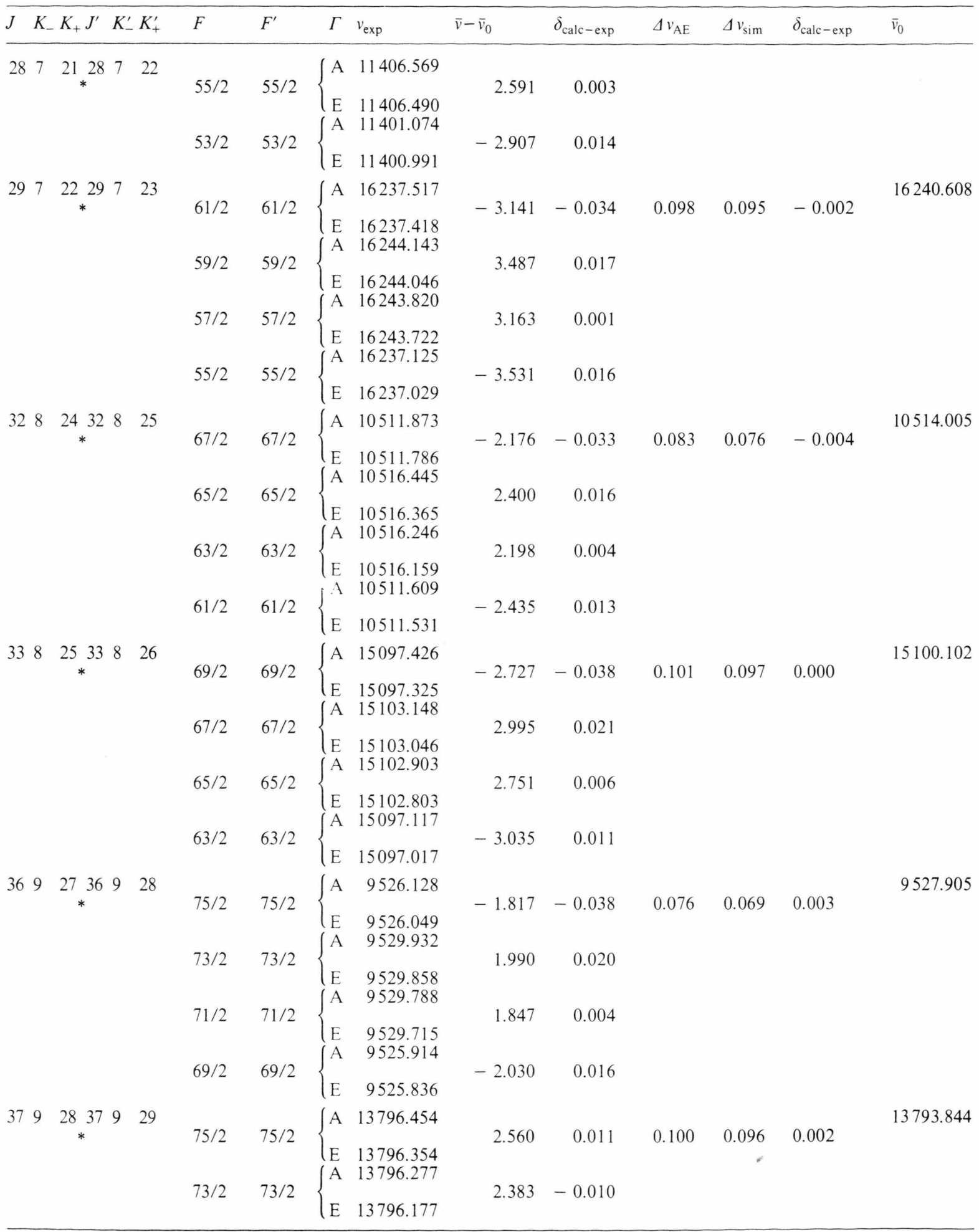


Table 2. Measured transitions of $\mathrm{CH}_{3} \mathrm{C}^{81} \mathrm{Br}=\mathrm{CH}_{2} . \Gamma$ : symmetry species, $v_{\text {exp }}$ : experimental frequency, $\bar{v}$ : arithmetic mean of the frequencies belonging to the same hfs transition and split by internal rotation, $\bar{v}_{0}:$ see text, $\bar{v}-\bar{v}_{0}:$ measured hfs shift, $\Delta v_{\mathrm{AE}}$ : experimental torsional splitting, $\Delta v_{\mathrm{sim}}$ : corrected torsional splitting, B: measured by Benz et al. [1], *: not used for quadrupole hfs analysis, nr: not resolved; frequencies, splittings and deviations in $\mathrm{MHz}$.

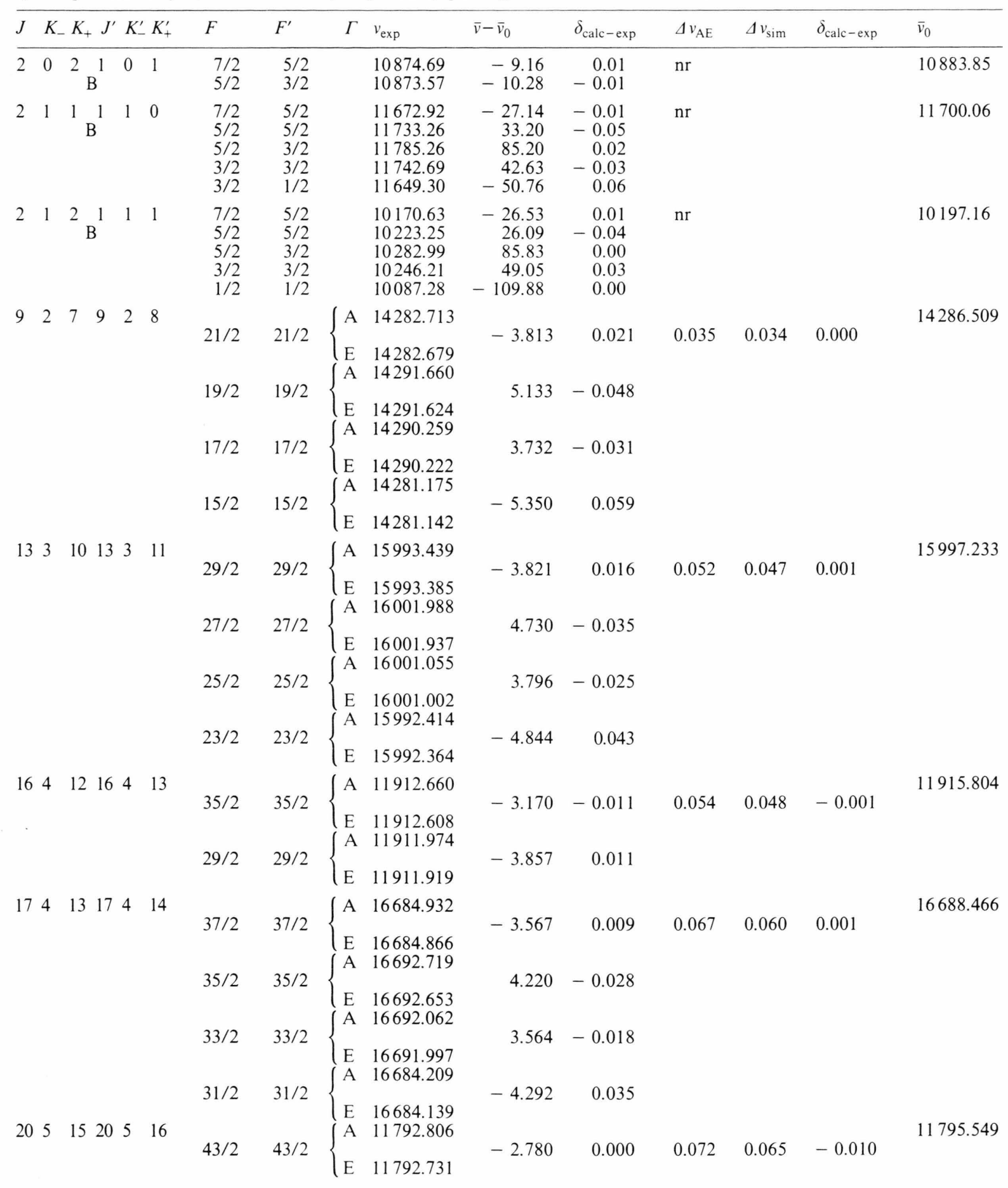


Table 2 (continued).

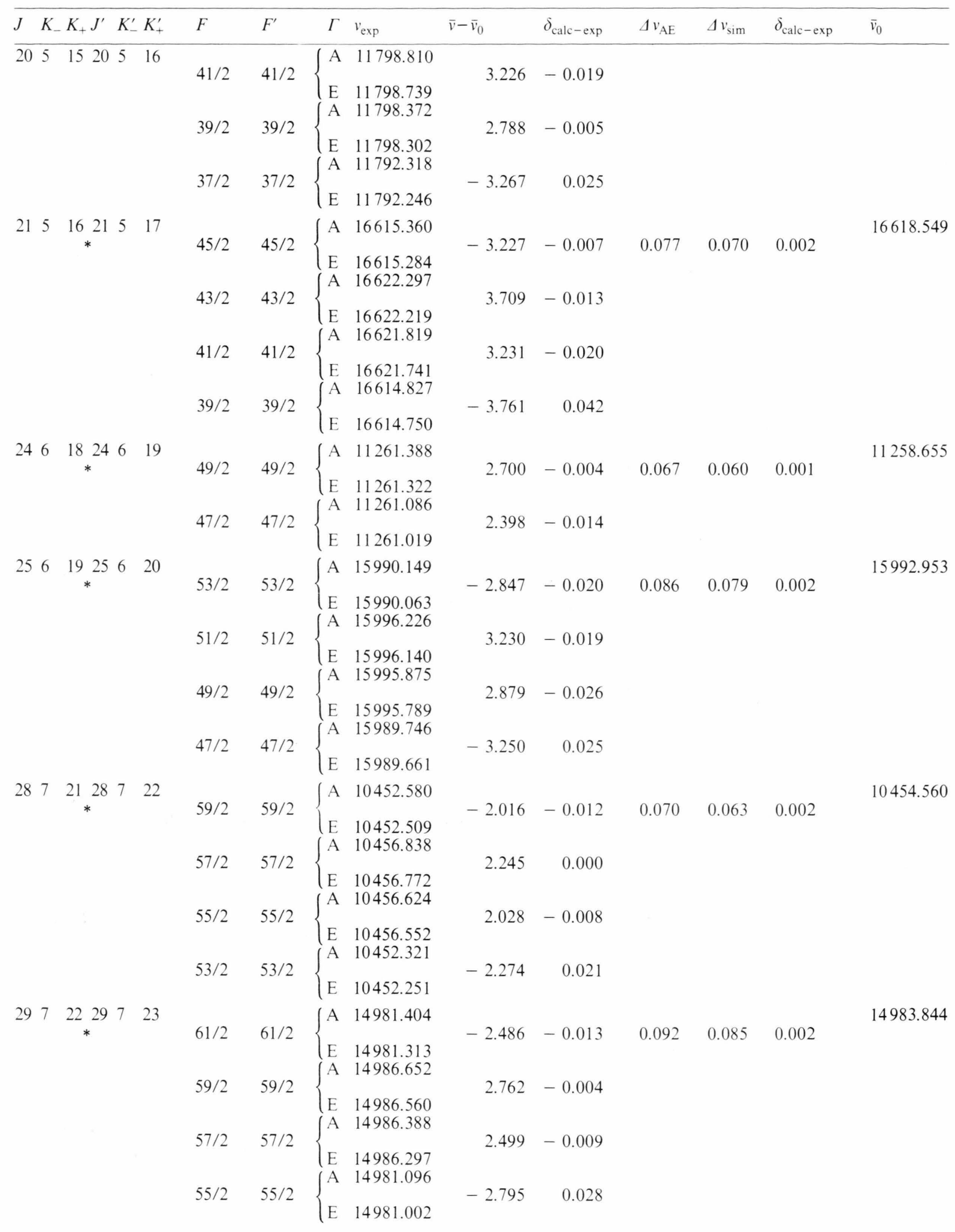


Table 2 (continued).

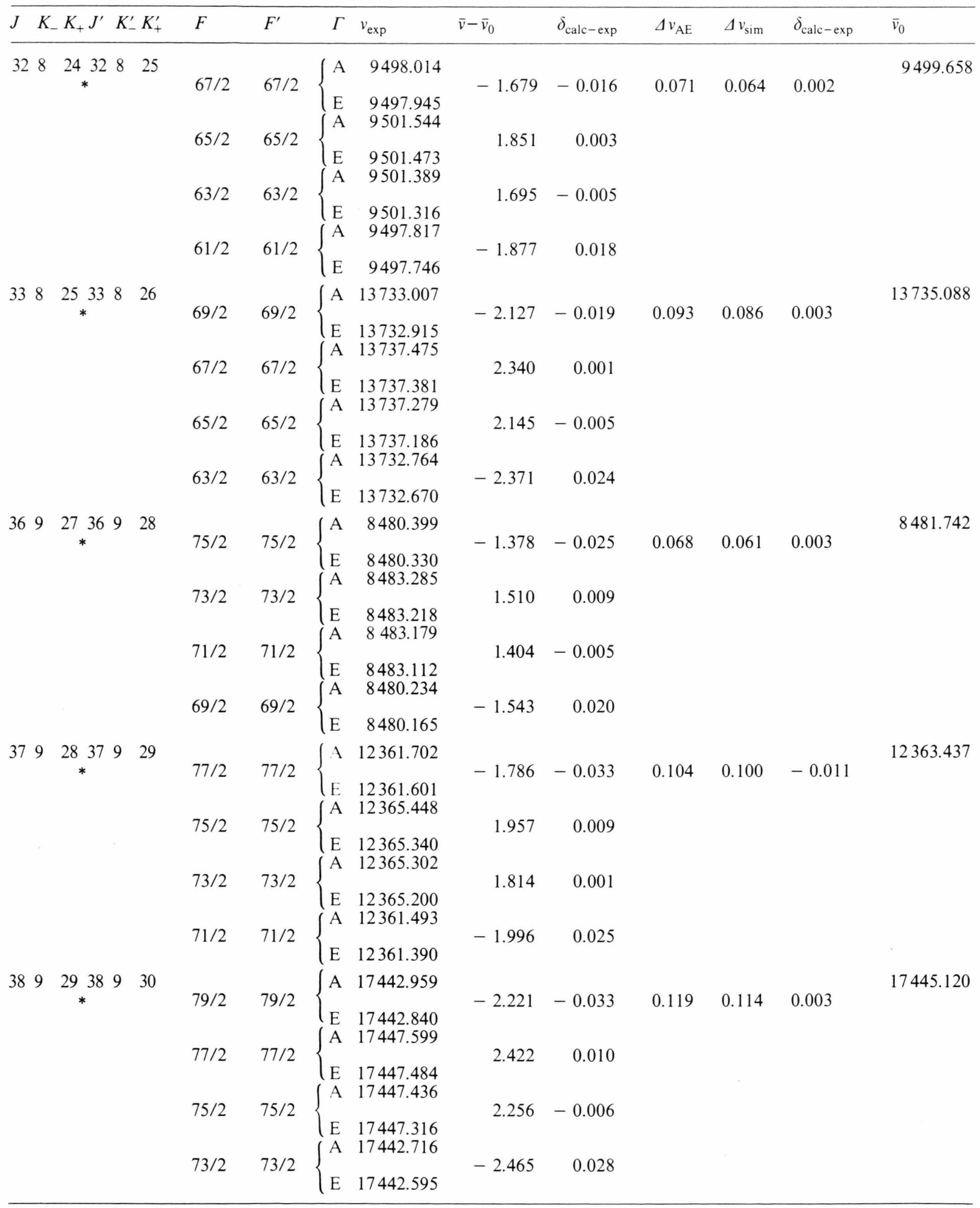


Table 3. Rotational [MHz], centrifugal distortion [kHz] and bromine-hfs [MHz] constants of 2-bromopropene. $N$ : number of lines, $\sigma$ : standard deviation of the fit [kHz], $\overline{\Delta v_{\text {exp }}}$ : mean experimental hfs splitting [MHz]; standard errors in units of the last digit in brackets. The centrifugal distortion constants $D_{J}^{\prime}, D_{J K}^{\prime}, D_{K}^{\prime}$ and $R_{6}^{\prime}$ were assumed to be zero.

\begin{tabular}{|c|c|c|c|c|c|c|c|c|c|c|}
\hline & $\mathrm{CH}_{3} \mathrm{C}^{79} \mathrm{Br}=\mathrm{CH}_{2}$ & Correlati & on matrix & & & $\mathrm{CH}_{3} \mathrm{C}^{81} \mathrm{Br}=\mathrm{CH}_{2}$ & Correlati & on matrix & & \\
\hline $\begin{array}{l}A \\
B \\
C \\
\delta_{J}^{\prime} \\
N \\
\sigma\end{array}$ & $\begin{array}{l}9256.00(10) \\
3138.011(26) \\
2375.879(26) \\
0.14557(71) \\
21 \\
175\end{array}$ & $\begin{array}{r}1.00 \\
0.38 \\
0.11 \\
-0.83\end{array}$ & $\begin{array}{r}1.00 \\
0.96 \\
-0.10\end{array}$ & $\begin{array}{l}1.00 \\
0.10\end{array}$ & 1.00 & $\begin{array}{l}9255.942(78) \\
3112.878(18) \\
2361.434(18) \\
0.14304(43) \\
18 \\
124\end{array}$ & $\begin{array}{r}1.00 \\
0.38 \\
0.08 \\
-0.81\end{array}$ & $\begin{array}{r}1.00 \\
0.95 \\
-0.11\end{array}$ & $\begin{array}{l}1.00 \\
0.11\end{array}$ & 1.00 \\
\hline $\begin{array}{l}\chi_{+} \\
\chi_{-} \\
N \\
\sigma \\
\Delta v_{\exp } \\
\chi_{a a} \\
\chi_{b b} \\
\chi_{c c}\end{array}$ & $\begin{array}{l}-534.474(53) \\
-38.222(59) \\
12 \\
29 \\
26.591 \\
534.474(53) \\
-286.348(56) \\
-248.126(56)\end{array}$ & $\begin{array}{l}1.000 \\
0.040\end{array}$ & 1.000 & & & $\begin{array}{c}-446.653(71) \\
-31.546(89) \\
8 \\
36 \\
29.760 \\
446.653(71) \\
-239.100(80) \\
-207.554(80)\end{array}$ & $\begin{array}{l}1.000 \\
0.008\end{array}$ & 1.000 & & \\
\hline
\end{tabular}

Table 4. Internal rotation parameters of 2-bromopropene $w_{1}(s)$ : Fourier coefficient, $\Varangle(a, i), \Varangle(b, i)$ : angle between the inertia axis $a$ resp. $b$ and the internal rotation axis $i, I_{\alpha}$ : moment of inertia of the methyl group, $N$ : number of lines, $(a, b)$ : correlation between $a$ and $b, \sigma$ : standard deviation of the fit, $\Delta v_{\mathrm{AE}}$ : mean experimental torsional splitting, $s$ : reduced barrier height, $V_{3}$ : barrier to internal rotation, $F$ : reduced rotational constant of the internal rotation, $\lambda_{a}, \lambda_{b}$ : direction cosines of $\Varangle(a, i)$ and $\Varangle(b, i)$; standard errors in units of the last digit in brackets, fixed values in square brackets, derived parameters below the line.

\begin{tabular}{lcc}
\hline & $\mathrm{CH}_{3} \mathrm{C}^{79} \mathrm{Br}=\mathrm{CH}_{2}$ & $\mathrm{CH}_{3} \mathrm{C}^{81} \mathrm{Br}=\mathrm{CH}_{2}$ \\
\hline$w_{1}(s)$ & $-4.13\left(69 \cdot 10^{-6}\right.$ & $-4.3(11) \cdot 10^{-6}$ \\
$\nless(a, i)\left[{ }^{\circ}\right]$ & $57.8(34)$ & $56.9(48)$ \\
$I_{\chi}\left[\mathrm{amu} \AA^{2}\right]$ & {$[3.167]$} & {$[3.167]$} \\
$N$ & 12 & 15 \\
$\left(w_{1}(s), \Varangle(a, i)\right)$ & 0.998 & 0.998 \\
$\sigma[\mathrm{kHz}]$ & 3 & 5 \\
$\Delta v_{\mathrm{AE}}[\mathrm{kHz}]$ & 73 & 69 \\
\hline$s$ & $72.8(14)$ & $72.4(21)$ \\
$V_{3}[\mathrm{cal} / \mathrm{mol}]$ & $2571(49)$ & $2558(74)$ \\
$F[\mathrm{GHz}]$ & 164.64 & 164.72 \\
$\nless(b, i)\left[{ }^{\circ}\right]$ & $32.2(34)$ & $33.1(48)$ \\
$i_{a}$ & $0.533(51)$ & $0.546(72)$ \\
$i_{b}$ & $0.846(30)$ & $0.838(43)$ \\
\hline
\end{tabular}

lines up to $J=20$ with a program diagonalising the Hamiltonian matrix [6]. Lines with higher $J$-values could not be included into the fit because of computer time and core capacity. The off-diagonal tensor element $\chi_{a b}$ could not be determined, since the measured lines are not sensitive enough to this parameter. We did not follow the $\chi_{a b}$ variation procedure reported in [1].
The line centres $\bar{v}_{0}$ of the hfs patterns were calculated by first averaging the internal rotation splittings (frequencies $\bar{v}$ ) and by secondly applying the hfs shifts predicted for lines up to $J=20$ by means of direct diagonalisation and for higher $J$ values according to first order theory with the constants of Table 3 . These frequencies $\bar{v}_{0}$ were the input for the centrifugal distortion analysis.

The results of the bromine quadrupole hfs and the centrifugal distortion analysis are given in Table 3.

For the internal rotation analysis we took the mean internal rotation splitting of the hfs components for each rotational line. This is equivalent to the approximation that nuclear quadrupole coupling and internal rotation do not interact.

As we noticed [7] that the spacings of narrowly split lines by the power spectra of MWFT are displayed slightly too large, we corrected the frequencies. From many line shape analyses of $\mathrm{CH}_{3} \mathrm{C}^{35} \mathrm{Cl}=\mathrm{CH}_{2}$ and $\mathrm{CH}_{3} \mathrm{C}^{79} \mathrm{Br}=\mathrm{CH}_{2}$ we got the same empirical dependence of the correction as a function of the splitting. This empirical correction was also applied to $\mathrm{CH}_{3} \mathrm{C}^{81} \mathrm{Br}=\mathrm{CH}_{2}$. The corrected splittings ( $\Delta v_{\text {sim }}$ of Tables 1 and 2$)$ are the basis for the internal rotation analysis.

The analysis was performed by the internal axis method (IAM) with a program by Woods $[8,9]$ which was modified $[10,11]^{*}$. The rotational constants of Table 3 were used. An attempt to fit the potential parameter $w_{1}(s)$, the angle $\Varangle(a, i)$ be-

* Programs DDIRFI, FIDIC, and F05MOD. 
Table 5. Comparison of potential barriers $V_{3}$ of 2-halopropenes $\mathrm{CH}_{3} \mathrm{CX}=\mathrm{CH}_{2} . I_{\alpha}=3.167$ amu $\AA^{2}$ was assumed [12]. Further assumption in square brackets, standard deviations in brackets.

\begin{tabular}{lccccc}
\hline$X$ & $F$ & ${ }^{35} \mathrm{Cl}$ & ${ }^{37} \mathrm{Cl}$ & ${ }^{79} \mathrm{Br}$ & ${ }^{81} \mathrm{Br}$ \\
\hline$V_{3}[\mathrm{cal} / \mathrm{mol}]$ & $2407.3(37)$ & $2568(10)$ & $2582(12)$ & $2571(49)$ & $2558(74)$ \\
$\nless(a, i)\left[^{\circ}\right]$ & {$[3.03]$} & $59.78(84)$ & $61.28(108)$ & $57.8(34)$ & $56.9(48)$ \\
$F[\mathrm{GHz}]$ & 170.43 & 165.91 & 165.69 & 164.64 & 164.72 \\
$S$ & $65.84(10)$ & $72.15(29)$ & $72.63(35)$ & $72.8(14)$ & $72.4(21)$ \\
\hline
\end{tabular}

tween the $a$ - and the internal rotation axis and the moment of inertia $I_{\alpha}$ of the methyl group resulted in an unreasonably high $I_{\alpha}=3.32 \mathrm{amu} \AA^{2}$ and $I_{\alpha}=$ $3.69 \mathrm{amu} \AA^{2}$ for the ${ }^{79} \mathrm{Br}$ - and ${ }^{81} \mathrm{Br}$-species. So we decided to assume $I_{\alpha}=3.167 \mathrm{amu} \AA^{2}$ like in the analysis of 2-chloropropene [12].

The results of the internal rotation analysis are given in Table 4. It should be mentioned that the barriers $V_{3}$ and the angles $\Varangle(a, i)$ of both isotopes coincide within the error limits.

To check the values of the angle $\Varangle(a, i)$, we performed tentative $r_{0}$-structure calculations. Unfortunately the information by the known six rotational constants is limited. So we transferred the structural data of 2-chloropropene of Tab. $\mathrm{V}$ of [13] and fitted the bond length $\overline{\mathrm{CBr}}$ and the angle $\Varangle \mathrm{CCBr}$. The angle of an internal rotation axis perpendicular to the plane of the three methyl hydrogen atoms with respect to the $a$-axis is roughly $62^{\circ}$ for both isotopes. Considering the inherent errors the agreement is satisfactory.

We also repeated the internal rotation analyses for 2-chloropropene $\mathrm{CH}_{3} \mathrm{C}^{x} \mathrm{Cl}=\mathrm{CH}_{2}$ with $x=35$ and 37 using corrected torsional splittings $\Delta v_{\text {sim }}$. The values of Table 5 of [12] thereupon only change within the error limits.

[1] H. P. Benz, A. Bauder, and Hs. H. Günthard, J. Mol. Spectr. 21, 165 (1966).

[2] G. Bestmann, H. Dreizler, H. Mäder, and U. Andresen, Z. Naturforsch. 35 a, 392 (1980).

[3] G. Bestmann and H. Dreizler, Z. Naturforsch. 37 a, 58 (1982).

[4] B. P. van Eijck, J. Mol. Spectr. 53, 246 (1974).

[5] V. Typke, J. Mol. Spectr. 63, 170 (1976); program ZFAP4 by V. Typke (1982).

[6] D. Hübner and M. Stolze, programs NQ20A and NQ20S, diplom theses, Kiel 1980.

[7] E. Fliege and H. Dreizler, Z. Naturforsch. 39 a, (1984).
Presently the methyl torsion analyses of the 2halopropenes $\mathrm{CH}_{3} \mathrm{CX}=\mathrm{CH}_{2}$ with $\mathrm{X}=\mathrm{F}[14],{ }^{35} \mathrm{Cl}$, ${ }^{37} \mathrm{Cl}[12],{ }^{79} \mathrm{Br}$, and ${ }^{81} \mathrm{Br}$ [this paper] are available. As the PAM-analysis for $\mathrm{CH}_{3} \mathrm{CF}=\mathrm{CH}_{2}$ given in Table 5 of [14] is based on $I_{\alpha}=3.11 \mathrm{amu} \AA^{2}$ which was taken from propylene, we repeated for comparison the analysis with $I_{\alpha}=3.167$ amu $\AA^{2}$ and $\Varangle(a, i)=3.03^{\circ}$. Without the assumption of $\Varangle(a, i)$ from the structure Fig. 1 of [14] the fit diverged. The result is given in Table 5. With more precise measurements as they are possible nowadays, the torsional analysis for 2-fluoropropene could most probably be improved.

There is a substantial difference of the $V_{3}$-values between the fluorine and the other halogen compounds. This has already been stated in [1], but the values of Table 5 stem all from an analysis of the ground state torsional fine structure carried out by the same method (IAM) under equivalent assumptions.

We thank the members of our group and especially Dipl.-Phys. G. Bestmann for help and the Deutsche Forschungsgemeinschaft and Fond der Chemie for funds.

* The line $28_{20.8}-28_{20,9}$ was omitted.

[8] R. C. Woods, J. Mol. Spectr. 21, 4 (1966).

[9] R. C. Woods, J. Mol. Spectr. 22, 49 (1967).

[10] J. Meier, Dissertation 4611, ETH Zurich 1970.

[11] Modified by H. Lutz and E. Fliege, Kiel.

[12] E. Fliege and H. Dreizler, Z. Naturforsch. 38 a, 1231 (1983).

[13] W. Good, R. J. Conan, jr., A. Bauder, and Hs. H. Günthard, J. Mol. Spectr. 41, 381 (1972).

[14] L. Pierce and J. M. O'Reilly, J. Mol. Spectr. 3, 536 (1959). 